\title{
LIFT on Keck: evaluation of performance and experiments towards an on-sky validation
}

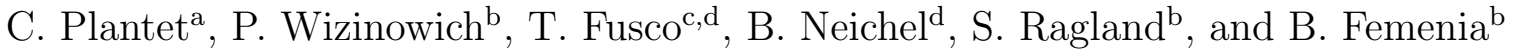 \\ ${ }^{a}$ INAF - Osservatorio di Arcetri, 50125 Firenze, Italy \\ ${ }^{\mathrm{b}}$ W. M. Keck Observatory, 65-1120 Mamalahoa Hwy., Kamuela, HI 96743, USA \\ ${ }^{\circ}$ Onera, the French Aerospace Lab, 92322 Chatillon, France \\ dAix Marseille Universite, CNRS, LAM (Laboratoire dAstrophysique de Marseille) UMR 7326, \\ 13388 Marseille, France
}

\begin{abstract}
Wavefront sensing in the near infrared has become an attractive option with the advent of new low-noise infrared detectors, such as the SAPHIRA (Selex) APD array. The performance improvements obtained with the H2RGbased Keck I near-infrared tip-tilt sensor is motivating the implementation of a near-infrared low-order sensor for Keck II. The recently proposed focal plane sensor algorithm LIFT could fulfill this role. We show here an analysis of performance, demonstrating that LIFT would provide a significant gain $(\sim 1$ magnitude) over the current tip/tilt sensor at low flux, as well as the first experimental validation of LIFT on Keck with a calibration source.
\end{abstract}

Keywords: Adaptive optics, Wavefront sensing, Infrared, Keck, LIFT

\section{INTRODUCTION}

Laser-assisted adaptive optics relies on natural guide stars for the estimation of low orders. ${ }^{1-5}$ Infrared wavefront sensors, compared to visible ones, provide some advantages for this application: lower wavefront distortion, hence better sensitivity, and access to colder stars or stars behind dust clouds, hence higher sky coverage. Moreover, cold stars of type $\mathrm{K}$ or $\mathrm{M}$, which emit more in the near infrared than in visible light (2 to 3 magnitudes difference $\left.{ }^{6}\right)$, represent a great majority of main-sequence stars. ${ }^{7}$ However, visible wavefront sensors have been preferred until now because of the difference in noise level between visible and infrared detectors. Recently, a H2RG-based camera was implemented with the Keck I laser guide star adaptive optics system to work as a nearinfrared ( $\mathrm{H}$ and/or Ks band) tip-tilt sensor. ${ }^{8}$ Low noise is achieved by multiple reads of small regions of interest. The on-sky results have been excellent and have motivated a proposal to implement a near-infrared low-order wavefront sensor on Keck II. The Keck II sensor will be based on the recent advances with $\mathrm{HgCdTe}$ avalanche photodiodes arrays, such as the SAPHIRA (Selex) detector, ${ }^{9}$ which offers very low noise (1 to few electrons), and thus wavefront sensing at low flux in the near infrared. The recently developed low-order wavefront sensor algorithm LIFT $^{10}$ is considered for this application. It is very simple to set up, as it makes its estimation from a single astigmatic focal image. Previous studies have shown that LIFT has good noise propagation properties, comparable to a non-modulated pyramid, for the estimation of tip/tilt and focus. ${ }^{11}$ It was validated in the lab ${ }^{11}$ and in open loop on sky with the Gemini South MCAO system GeMS. ${ }^{12}$ In this paper, we theoretically study the performance of LIFT for an application on Keck, and compare it to the current near-infrared tip/tilt sensor. We also report on tests performed to prepare an implementation of LIFT on the Keck telescopes.

\section{PRELIMINARY STUDY}

The goal of this study is to predict LIFT's performance for the tip/tilt/focus estimation on Keck, and make sure it provides a significant gain compared to the H2RG-based tip/tilt sensor, TRICK. The first step is a conceptual design that will help us know the background flux to be expected. We then compute the error due to noise and high order effects to determine the best sampling to use. Finally, we compare LIFT's performance with the theoretical error on TRICK.

Further author information:

C.P.: E-mail: plantet@arcetri.astro.it 


\subsection{Conceptual design}

LIFT only requires an imaging camera and an astigmatism offset. Figure 1 shows a conceptual design, assuming the astigmatism offset is inserted before the dewar. It consists of an afocal objective which focuses the beam with a f-number corresponding to the desired sampling. The value of the imaging f-number is discussed in the next paragraph. The detector considered here is a SAPHIRA from Selex. Its characteristics, taken from First Light Imaging's C-RED datasheet, are listed in Tab. 1.



Figure 1: LIFT preliminary design.

Table 1: Selex SAPHIRA characteristics.

\begin{tabular}{|c|c|}
\hline Pixel size & $24 \mu \mathrm{m}$ \\
\hline Detector size & $320 \times 256$ pixels \\
\hline Read-out noise (gain 1) & $24 \mathrm{e}^{-}$ \\
\hline Quantum efficiency & $70 \%$ \\
\hline Dark current & $500 \mathrm{e}^{-} / \mathrm{s}$ (worst case assumption) \\
\hline Excess noise factor & 1.25 \\
\hline
\end{tabular}

\subsection{Optimal plate scale and performance}

We compute LIFT's performance, in terms of noise propagation and sensitivity to high order effects, for different plate scales (i.e. angular size of the pixel on sky) in order to determine the optimal imaging f-number. The considered plate scales are: 50 mas (TRICK's plate scale), 32 mas (Nyquist/2 sampling at the cut-on wavelength of $\mathrm{H}$ band), 22 mas (Nyquist sampling at the central wavelength of Ks band) or 17 mas (Nyquist sampling at the central wavelength of $\mathrm{H}$ band). The variance of estimation error is given by: ${ }^{10}$

$$
\sigma_{\text {est }}^{2}=\frac{\alpha F}{\eta n_{p h}}+\beta\left(\frac{\sigma_{\text {det }}}{\eta n_{p h}}\right)^{2}
$$

with $F$ the excess noise factor, $\eta$ the quantum efficiency, $n_{p h}$ the detected flux and $\sigma_{\text {det }}$ the equivalent detector noise. $\alpha$ and $\beta$ are the noise propagation coefficients for photon noise and detector noise respectively. These coefficients can be computed at the diffraction limit for each wavefront mode via the method described in Plantet et al. ${ }^{13}$ For LIFT, when not at the diffraction limit, $\alpha$ is scaled by $1 / S R$ and $\beta$ is scaled by $1 / S R^{2},{ }^{14}$ with $S R$ the Strehl ratio. The equivalent detector noise is computed as follows:

$$
\sigma_{\text {det }}=\frac{\sqrt{\sigma_{\text {ron }}^{2}+F G^{2} i_{\text {dark }} T_{i n t}+F G^{2} T_{i n t} \eta\left(F_{p i x, t h}+F_{p i x, s k y}\right)}}{G}
$$

with $\sigma_{\text {ron }}^{2}$ the read-out noise variance, $G$ the avalanche photodiode gain, $T_{i n t}$ the exposure time and $F_{p i x, t h}$ and $F_{p i x, s k y}$ the thermal and sky background fluxes respectively. We consider a gain $G=30$ here. The thermal 
background flux per pixel is equal to:

$$
F_{p i x, t h}=\left(1-T_{t e l}\right) \frac{\pi L_{B B}}{4 N_{o b j}^{2}} l_{p i x}^{2}
$$

with $T_{t e l}$ the total throughput from the primary mirror to the cryostat ( $55 \%$ here), $L_{B B}$ the black body radiance, $N_{o b j}$ the afocal objective f-number and $l_{p i x}$ the pixel size. The sky background flux per pixel is:

$$
F_{p i x, s k y}=T_{t e l} F_{0} \times \frac{\pi}{4} D^{2} \times 10^{-\frac{L_{S}}{2.5}} \times l_{p i x}^{2}
$$

with $F_{0}$ the zero magnitude flux in $\mathrm{ph} / \mathrm{s} / \mathrm{m}^{2}, D$ the telescope diameter $(10 \mathrm{~m})$ and $L_{S}$ the sky radiance expressed in magnitudes. We consider $L_{S}=13.93 \mathrm{mag} / \operatorname{arcsec}^{2}$ in $\mathrm{H}$ band and $L_{S}=15.2 \mathrm{mag} / \mathrm{arcsec}^{2}$ in $\mathrm{Ks}$ band (values from Lianqi Wang at the Thirty Meter Telescope). For a star of magnitude $m$, the number of photons $n_{p h}$ is:

$$
n_{p h}=T_{t e l} F_{0} \times \frac{\pi}{4} D^{2} \times 10^{-\frac{m}{2.5}} \times T_{i n t}
$$

We have computed $\alpha$ and $\beta$ for the different plate scales in order to have an estimation error as a function of magnitude. Besides, we performed closed loop simulations to evaluate the sensitivity to high order effects: at low Strehl ratios, the small phase approximation becomes invalid, and we make an additional error. These simulations rely on phase residuals in a laser-assisted adaptive optics system, computed from a Fourier analysis. ${ }^{15}$ The parameters of these simulations are listed in Tab. 2, and the Strehl ratios (tip/tilt focus removed) for each considered case are given in Tab. 3. Tip/tilt and focus were replaced to correspond to the turbulence and were corrected by LIFT at $1000 \mathrm{~Hz}$ without noise. LIFT estimates here 14 modes with a Maximum A Posteriori estimator, using the true variance of each mode coefficient as an a priori. The residual error on tip/tilt and focus was averaged over 10 occurrences. We find that the error variance can be expressed as $\sigma_{e r r}^{2}=a \times \exp (-b \times \sqrt{S R})$ (Fig. 2), with $a$ and $b$ positive scalars depending on the sampling and the sensed mode. We use this formula to derive the error due to high orders, which we add to the noise error, and plot the resulting wavefront error in $\mathrm{nm} \mathrm{rms}$ as a function of magnitude in Fig. 3, for a Strehl ratio of $25 \%$ in Ks band (8.5\% in $\mathrm{H}$ ).

Table 2: Closed loop simulation parameters.

\begin{tabular}{|c|c|}
\hline Telescope diameter & $10.95 \mathrm{~m}$ \\
\hline Subaperture size & $0.5625 \mathrm{~m}$ \\
\hline Correction cadency & $1 \mathrm{kHz}$ \\
\hline Correction delay & $3 \mathrm{~ms}$ \\
\hline Correction gain & 0.3 \\
\hline Number of turbulent layers & 7 \\
\hline Layers' altitudes (m) & $0,500,1000,2000,4000,8000,16000$ \\
\hline$C_{n}^{2}$ profile (normalized in energy) & $0.517,0.119,0.063,0.061,0.105,0.081,0.054$ \\
\hline Mean wind speed & $9.5 \mathrm{~m} / \mathrm{s}$ \\
\hline Seeing & $0.3 " / 0.5 "$ \\
\hline Zenith angle & $30^{\circ}$ \\
\hline NGS separation & $0 ", 20 ", 40^{\prime \prime}$ \\
\hline NGS sensing wavelength & $2.15 \mu \mathrm{m}$ \\
\hline
\end{tabular}


Table 3: Strehl ratio for the different cases (tip/tilt and focus removed).

\begin{tabular}{|c|c|c|}
\hline NGS separation & Strehl ratio (seeing =0.3") & Strehl ratio (seeing =0.5") \\
\hline $0 "$ & 0.68 & 0.37 \\
\hline $20 "$ & 0.56 & 0.24 \\
\hline $40 "$ & 0.44 & 0.15 \\
\hline
\end{tabular}

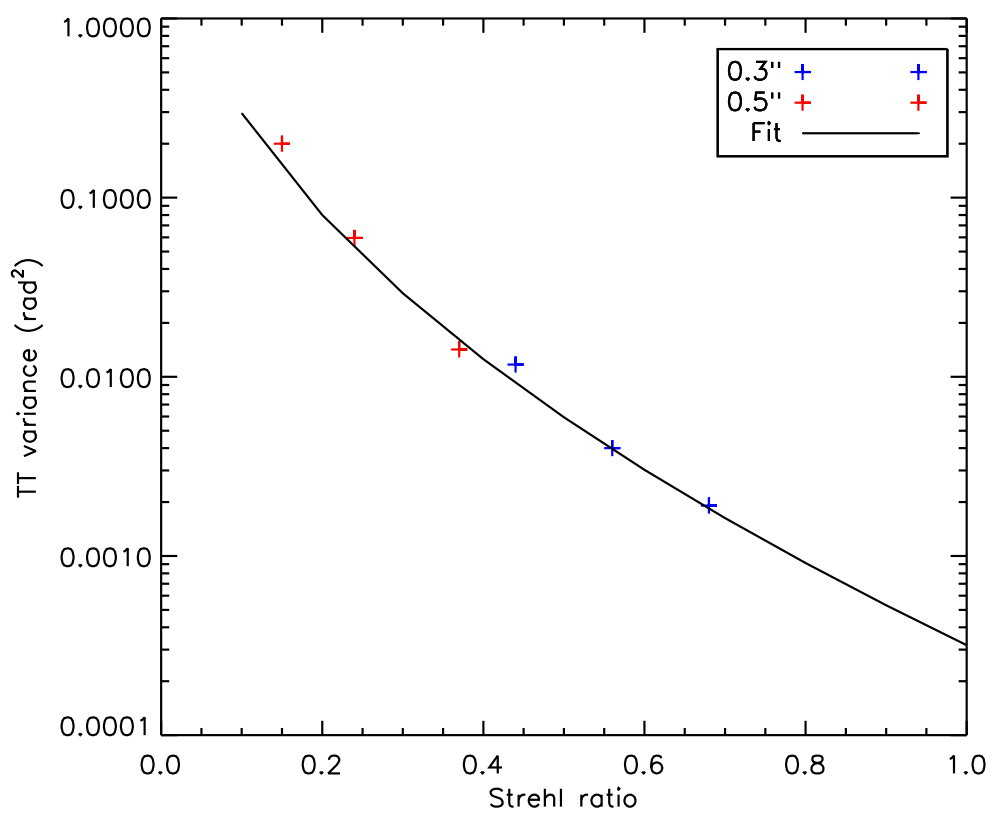

Figure 2: Residual variance on tip/tilt in closed loop for the different considered cases and for a sampling at 0.55 Nyquist. Each point correspond to a different separation from the axis of correction.



(a) Tip/tilt

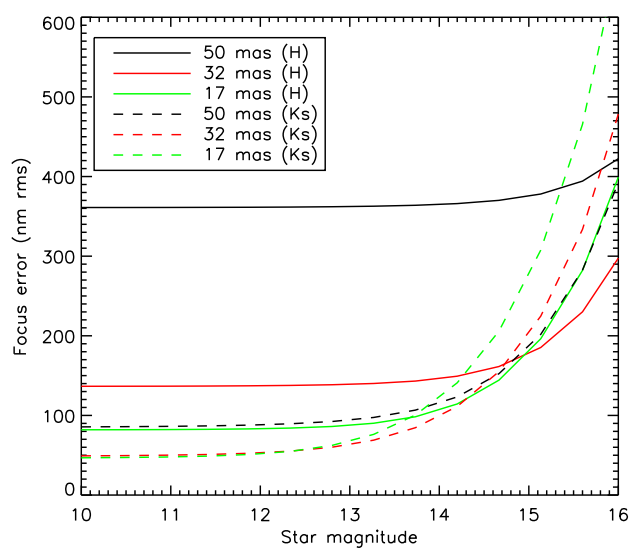

(b) Focus

Figure 3: LIFT's performance for different plate scales. Left: Tip/tilt estimation (100 $\mathrm{nm}=8.2 \mathrm{mas})$. Right: Focus estimation. The acquisition frequency is $300 \mathrm{~Hz}$ and the Strehl ratio is $25 \%$ in $\mathrm{Ks}$ band $(8.5 \%$ in $\mathrm{H})$. 
For the new system, the Ks band will not be used for wavefront sensing. In that case, the pixel scale offering the best trade-off is 17 mas. We thus choose this plate scale for LIFT on the Selex camera, and compare it to TRICK for the estimation of tip/tilt (Fig. 4a). For this comparison, only the noise error is taken into account. TRICK uses a classical center of gravity on $4 \times 4$ pixels. We evaluated its noise error from a computation of its Fisher coefficients. ${ }^{13}$ We also compare the resulting spot FWHM on the science camera from using TRICK or LIFT on the H2RG detector, taking into account the high order effects (Fig. 4b). We consider TRICK's tip/tilt estimation is impacted by high orders the same way as LIFT's estimation is. The spot FWHM is derived from the quadratic addition of the diffraction spot FWHM and the tip/tilt error in mas: $F W H M=\sqrt{F W H M_{d i f f}^{2}+\sigma_{T T}^{2}}$. When science is done in $\mathrm{H}$ band, the estimation is done in Ks band, and reciprocally.



(a) Noise error



(b) FWHM

Figure 4: Comparison of LIFT and TRICK for the estimation of tip/tilt. (a) Noise error comparison of TRICK (H2RG) with LIFT for plate scales of 17 mas (LIFT on Selex) and 50 mas (LIFT on H2RG). (b) Resulting FWHM for a plate scale of 50 mas, taking into account the high order effects (Centroid = TRICK). The black lines show the diffraction limit in $\mathrm{H}$ (lower line) and Ks (upper line). The acquisition frequency is adapted to the star magnitude and goes from $800 \mathrm{~Hz}$ (magnitude 10) to $100 \mathrm{~Hz}$ (magnitude 16). The Strehl ratio is $25 \%$ in Ks band $(8.5 \%$ in $\mathrm{H})$.

LIFT is less sensitive to noise than the centroid, especially at low flux, where detector noise dominates. Indeed, LIFT applies a weighting map on the data, which depends on the noise statistics: in detector noise regime, pixels far from the spot center are not taken into account. At high flux, the difference in error noise between LIFT and the centroid is negligible compared to the high orders error. However, at low flux, LIFT provides a gain of $\sim 1$ magnitude in terms of spot FWHM on the science camera. Besides, it provides a minimum FWHM on a wide range of flux (up to the 15th magnitude).

\section{EXPERIMENTS}

\subsection{Principle}

The goal of these tests is to validate the good estimation of low-order modes by LIFT in Keck's adaptive optics system. To do this, we first closed the loop on a calibration source (Fig 5). Then, by changing the reference slopes on the wavefront sensor (a Shack-Hartmann), we inserted an astigmatism offset on the DM, needed for LIFT's estimation, as well as ramps of Zernike modes.

We have also made experiments using only the non-common path aberrations on TRICK, that are dominated by astigmatism, as a phase offset. This would let us use LIFT on TRICK's camera without requiring any additional device to introduce the astigmatism. This procedure is of course preferable for on-sky operations. 
Finally, in order to prepare a first open-loop on-sky validation, we introduced a ramp of focus with a simulated turbulence corresponding to a Strehl ratio degradation down to $20 \%$. The simulated turbulence is done with random sets of Zernike modes (from astigmatism up to mode 45) on the DM. In that case, the focus is introduced by moving the calibration source in $\mathrm{Z}$.

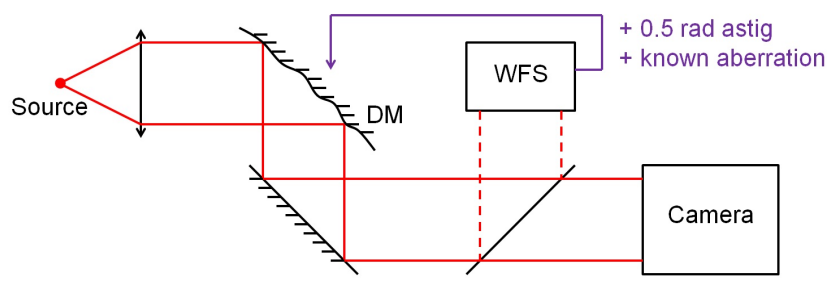

Figure 5: Principle of the experiment.

\subsection{Imaging model}

When using TRICK, we are dealing with very undersampled images ( 0.45 times Nyquist sampling in Ks band). To simulate them, we first compute a PSF at a sampling $k S \geqslant 1$ with $k$ a positive integer and $S$ the oversampling factor ( $S=1$ for Nyquist sampling). This PSF can be written:

$$
P S F_{0}(x, y)=h(x, y) \times I I I_{l_{p i x} / k}(x, y)
$$

with $h$ the PSF at infinite sampling and $I I I_{d}(x, y)$ the Dirac comb of period $d$. We then convolve this PSF to the pixel response (scaled by a factor $k$ ):

$$
P S F_{1}(x, y)=F T^{-1}\left\{F T\left[P S F_{0}(x, y)\right] \times \widetilde{f_{p i x}}(k u, k v)\right\}
$$

with $\widetilde{f_{p i x}}(u, v)$ the Fourier transform of the pixel response. Finally, we re-sample with the correct Dirac comb:

$$
P S F(x, y)=P S F_{1}(x, y) \times I I I_{l_{p i x}}(x, y)
$$

We consider here a Gaussian pixel response, with a full width at half maximum of 1 pixel. This is consistent with a previous characterization of similar detectors. ${ }^{16}$

\subsection{Results with TRICK}

\subsubsection{Linearity}

We plot in Fig. 6 the estimation of modes up to astigmatism with LIFT on TRICK, with the first procedure described in section 3.1. The images were taken in Ks band, and the astigmatism offset is null for tip/tilt and $170 \mathrm{~nm}(\sim 0.5 \mathrm{rad})$ for other modes. The tip/tilt is perfectly linear and matches the center of gravity estimation, taken as a reference, with a maximum error of 1.5 mas $(0.06 \mathrm{rad})$. The best focus estimation was obtained when considering an astigmatism of $0.35 \mathrm{rad}(120 \mathrm{~nm})$ in the model, meaning that we would have a scaling factor of 0.7 on astigmatism. The maximum error is then $30 \mathrm{~nm}$, or $0.09 \mathrm{rad}$ (with respect to the linear fit). The $45^{\circ}$ astigmatism is well estimated in positive values, with a maximum error of $13 \mathrm{~nm}$ (0.04 rad), though with a scaling factor of $\sim 0.6$. There is an inflection point around $-90 \mathrm{~nm}$ of inserted astigmatism, which reveals where the astigmatism offset is nulled, and even modes (e.g. focus, astigmatism...) are again undetermined. This means that we have a factor $\sim 0.53$ on the inserted astigmatism. In the end, when averaging the scaling factors $(0.53,0.6$ and 0.7$)$, the real factor seems to be around 0.6. Finally, the $0^{\circ}$ astigmatism estimation is slightly non-linear, but most amplitudes are correctly estimated. The maximum error is $50 \mathrm{~nm}(0.15 \mathrm{rad})$. The non-linearities on focus and $0^{\circ}$ astigmatism are most probably due to a model error. 


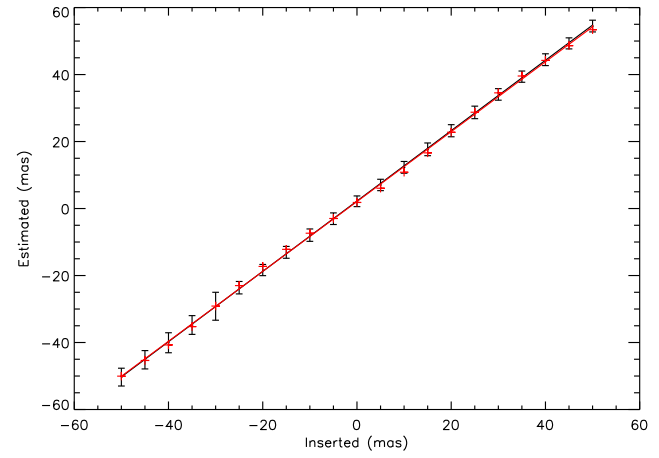

(a) Tilt

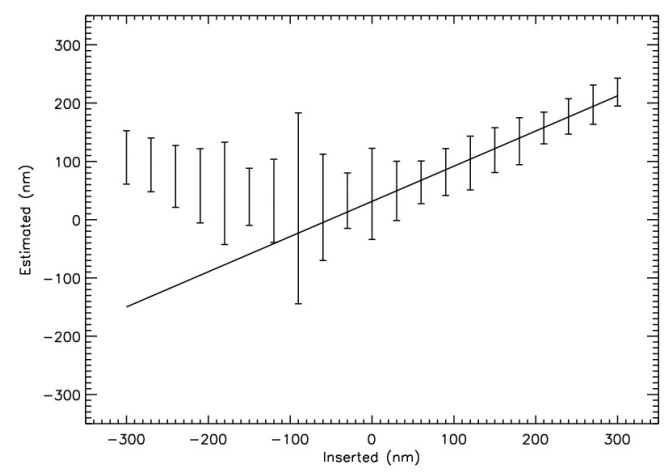

(c) Astig 45

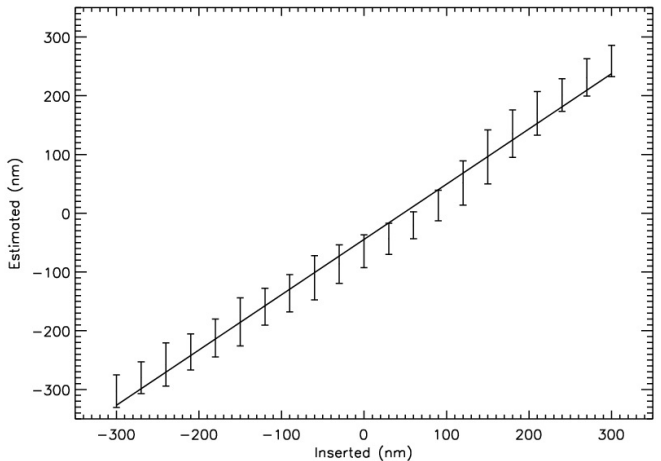

(b) Focus

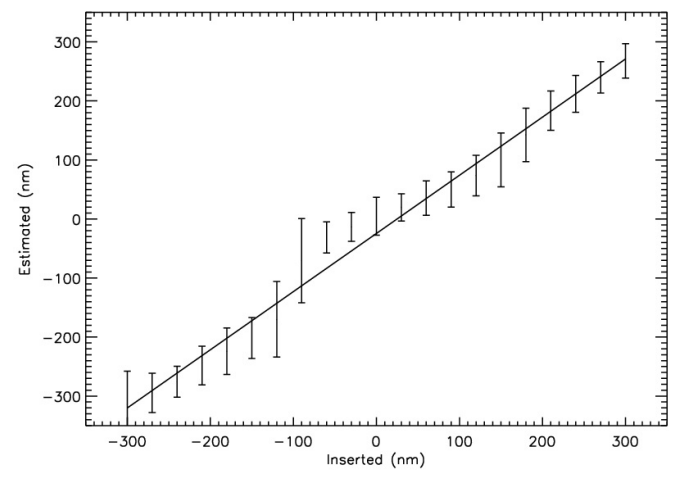

(d) Astig 0

Figure 6: Estimation of different aberration ramps with LIFT on TRICK in Ks band. The astigmatism offset of $170 \mathrm{~nm}(0.5 \mathrm{rad})$ is introduced with the DM. A $0.35 \mathrm{rad}$ astigmatism is considered in the model. The red plot in (a) is a center of gravity estimation. The images are sampled at 0.45 times Nyquist sampling.

We then took images with the astigmatism already present on TRICK's path. The estimation of focus and astigmatism ramps is plotted in Fig. 7. A first processing of the focus ramp gave us an estimate of the astigmatism offset: $50 \mathrm{~nm}$ of $45^{\circ}$ astigmatism and $140 \mathrm{~nm}$ of $0^{\circ}$ astigmatism, that is $\sim 150 \mathrm{~nm}$ of astigmatism in total $(0.43 \mathrm{rad})$. This astigmatism is used in the model and we obtain a satisfying linearity (15 $\mathrm{nm} \mathrm{rms}$ of error). We are thus able to use LIFT with the "natural" astigmatism present on TRICK's path, which is a great advantage on the operational point of view. One can notice that, in this case, we do not observe the inflection point present on Fig. 6 on the astigmatism offset. This is simply due to the fact that the offset is not solely on one astigmatism mode. 


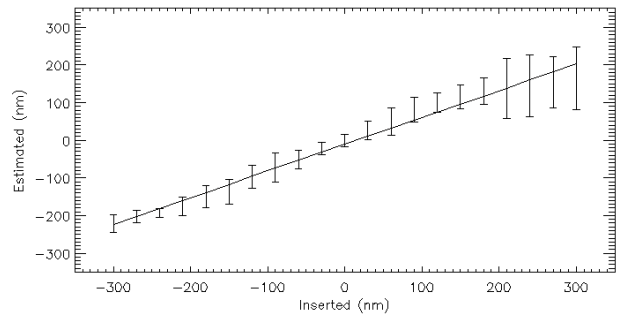

(a) Focus

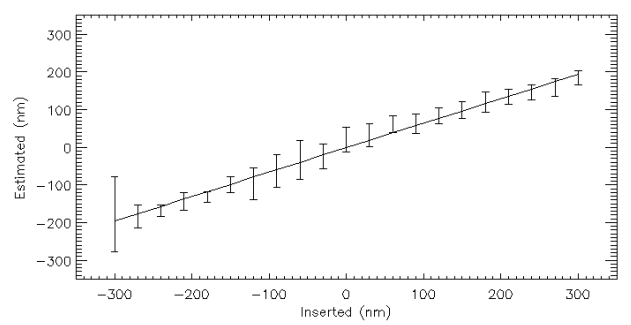

(b) Astig 45

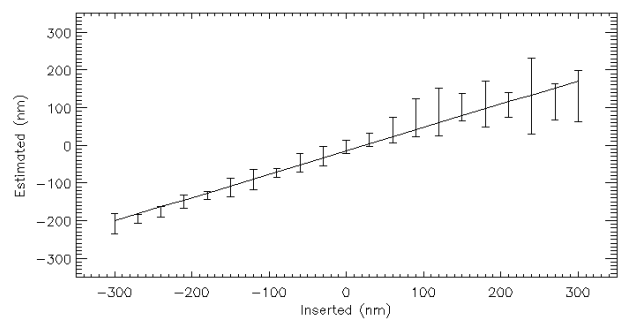

(c) Astig 0

Figure 7: Estimation of different aberration ramps with LIFT on TRICK in Ks band. The astigmatism offset comes from the non-common path aberrations of TRICK. The astigmatism in the model (derived from first estimations) is $50 \mathrm{~nm}$ at 45 and $140 \mathrm{~nm}$ at 0 . The images are sampled at 0.45 times Nyquist sampling.

These results demonstrate that we can estimate low orders up to astigmatism with LIFT at very low sampling $(<0.5$ Nyquist), and that no modification to the current hardware is needed to perform LIFT on TRICK's camera. The next step is to verify the ability of LIFT to make its estimation from a turbulent wavefront.

\subsubsection{Simulated turbulence}

Figure 8 shows the average estimation of a focus ramp with a simulated turbulence. For this test, we used 40 occurrences of Zernike modes, with an amplitude corresponding to a Strehl ratio of $20 \%$.

The average estimation is linear, with a slight offset at zero $(20 \mathrm{~nm})$, that is similar to the offset we had on previous curves. However, we have a lower slope, that is most probably due to the energy leaving the PSF core. As LIFT performs a weighting on the image, this loss of energy implies an under-estimation.

This result demonstrates the ability to perform an open-loop on-sky test, that would have the same procedure, with the natural turbulence instead of a simulated one. 


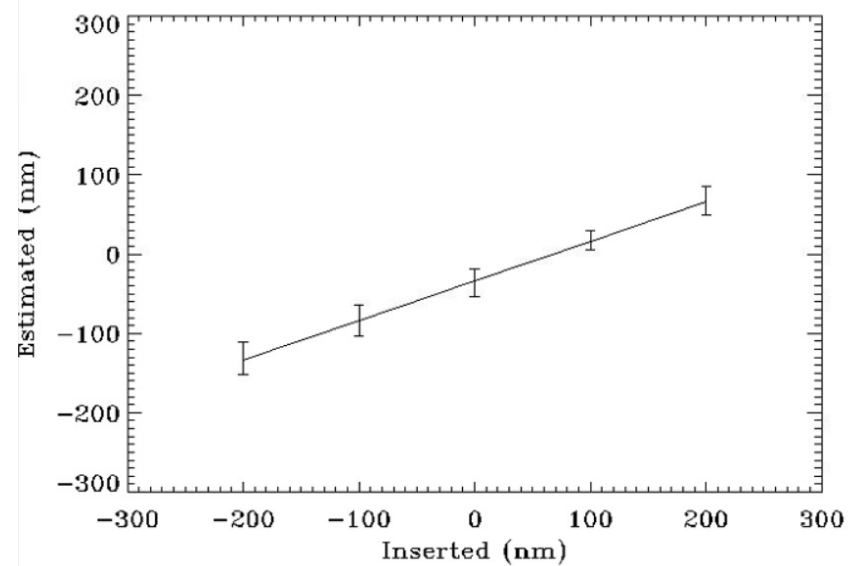

Figure 8: Average estimation of a focus ramp on TRICK with 40 occurrences of simulated turbulence. The turbulence is made by introducing random Zernike modes (from astigmatism to mode 45) on the DM and corresponds to a Strehl ratio of $20 \%$.

\section{CONCLUSION}

We have studied the performance of LIFT for an estimation of tip/tilt and focus on Keck telescopes. We have compared it to the current near-infrared tip/tilt sensor of Keck I, TRICK, and we have showed that it would provide a gain of $\sim 1$ magnitude in terms of spot FWHM at low flux, without changing the camera (H2RG) or the pixel scale (50 mas). Changing to a Selex camera and a 17 mas pixel scale would give a slightly better performance and could be considered for the future near-infrared sensor on Keck II. Moreover, LIFT would also provide a focus estimation, meaning that a slow focus sensor would not have to be implemented, though LIFT still has to be compared to the current slow focus sensor to verify this point.

We have also tested LIFT with Keck's calibration sources. We have shown that LIFT is able to estimate modes up to astigmatism at very low sampling (0.45 times the Nyquist sampling in Ks band), corresponding to the 50 mas plate scale on the H2RG camera. Besides, LIFT could be operationally performed on the H2RG camera without adding or modifying any physical element, thanks to the astigmatism offset present on TRICK's path. The implementation of LIFT on Keck I would thus be purely numerical. Finally, we have demonstrated the good estimation of focus in a simulated turbulence, and we are now ready for an on-sky validation in open loop, that will be followed by a final validation in closed loop.

\section{ACKNOWLEDGMENTS}

This work has been partially supported by the LabEx FOCUS ANR-11-LABX-0013 and by INAF (Research Grant DD 27). The W. M. Keck Observatory is operated as a scientific partnership among the California Institute of Technology, the University of California, and the National Aeronautics and Space Administration. The Observatory was made possible by the generous financial support of the W. M. Keck Foundation. The Keck I near-IR tip-tilt sensor was supported by the National Science Foundation under Grant No. AST-1007058 and by the Gordon and Betty Moore Foundation under Grant No. 4046.

\section{REFERENCES}

[1] Fusco, T., Meimon, S., Clenet, Y., Cohen, M., Paufique, J., and Schnetler, H., "ATLAS: the E-ELT laser tomographic adaptive optics system," Proc. SPIE 7736(77360D) (2010).

[2] Diolaiti, E. et al., "Towards the phase A review of MAORY, the multi-conjugate adaptive optics module for the E-ELT," Proc. AO4ELT (2007).

[3] Herriot, G., Hickson, P., Ellerbroek, B., Andersen, D., Davidge, T., Erickson, D., Powell, I., Clare, R., Gilles, L., Boyer, C., et al., "NFIRAOS: TMT narrow field near-infrared facility adaptive optics," in [Astronomical Telescopes and Instrumentation], 62720Q-62720Q, International Society for Optics and Photonics (2006). 
[4] Neichel, B. et al., "The Gemini MCAO System GeMS: nearing the end of a lab-story," Proc. SPIE 7736(773606) (2010).

[5] Wizinowich, P., Le Mignant, D., Bouchez, A. H., Campbell, R. D., Chin, J. C., Contos, A. R., van Dam, M. A., Hartman, S. K., Johansson, E. M., Lafon, R. E., et al., "The WM Keck Observatory laser guide star adaptive optics system: overview," Publications of the Astronomical Society of the Pacific 118(840), 297 (2006).

[6] Bessell, M., Castelli, F., and Plez, B., "Model atmospheres broad-band colors, bolometric corrections and temperature calibrations for O-M stars," Astronomy and astrophysics 333, 231-250 (1998).

[7] Ledrew, G., "The real starry sky," Journal of the Royal Astronomical Society of Canada 95, 32 (2001).

[8] Wizinowich, P., Smith, R., Biasi, R., Cetre, S., Dekany, R., Femenia-Castella, B., Fucik, J., Hale, D., Neyman, C., Pescoller, D., et al., "A near-infrared tip-tilt sensor for the Keck I laser guide star adaptive optics system," in [SPIE Astronomical Telescopes+ Instrumentation], 91482B-91482B, International Society for Optics and Photonics (2014).

[9] Feautrier, P., Gach, J.-L., and Wizinowich, P., "State of the art IR cameras for wavefront sensing using e-APD MCT arrays," in [AO4ELT4 Proceedings], (2015).

[10] Meimon, S., Fusco, T., and Mugnier, L. M., "LIFT: a focal-plane wavefront sensor for real-time low-order sensing on faint sources," Optics letters 35(18), 3036-3038 (2010).

[11] Plantet, C., Meimon, S., Conan, J.-M., and Fusco, T., "Experimental validation of LIFT for estimation of low-order modes in low-flux wavefront sensing," Optics Express 21, 16337-16352 (July 2013).

[12] Plantet, C., Meimon, S., Conan, J., Neichel, B., and Fusco, T., "On-sky validation of LIFT on GeMS," in [Proceedings of the Third AO4ELT Conference], Esposito, S. and Fini, L., eds., AO4ELT, INAF - Osservatorio Astrofisico di Arcetri, Firenze (2013). May 2013, Florence.

[13] Plantet, C., Meimon, S., Conan, J.-M., and Fusco, T., "Revisiting the comparison between the shackhartmann and the pyramid wavefront sensors via the fisher information matrix," Optics express 23(22), 28619-28633 (2015).

[14] Plantet, C., Meimon, S., Conan, J.-M., Neichel, B., and Fusco, T., "LIFT: analysis of performance in a laser assisted adaptive optics," in [SPIE Astronomical Telescopes+ Instrumentation], 91482D-91482D, International Society for Optics and Photonics (2014).

[15] Neichel, B., Fusco, T., and Conan, J.-M., "Tomographic reconstruction for wide-field adaptive optics systems: Fourier domain analysis and fundamental limitations," JOSA A 26(1), 219-235 (2009).

[16] Barron, N., Borysow, M., Beyerlein, K., Brown, M., Lorenzon, W., Schubnell, M., Tarlé, G., Tomasch, A., and Weaverdyck, C., "Subpixel response measurement of Near-Infrared detectors," Publications of the Astronomical Society of the Pacific 119(854), 466-475 (2007). 